\title{
A Cooperative Stochastic Differential Game of Transboundary Industrial Pollution between Two Asymmetric Nations
}

\author{
Yongxi Yi, Rongwei Xu, and Sheng Zhang \\ School of Economics \& Management, University of South China, Hengyang, China \\ Correspondence should be addressed to Yongxi Yi; yyx19999@126.com
}

Received 23 December 2016; Accepted 13 March 2017; Published 18 April 2017

Academic Editor: Huanqing Wang

Copyright (c) 2017 Yongxi Yi et al. This is an open access article distributed under the Creative Commons Attribution License, which permits unrestricted use, distribution, and reproduction in any medium, provided the original work is properly cited.

\begin{abstract}
Considering the fact that transboundary pollution control calls for the cooperation between interested parties, this paper studies a cooperative stochastic differential game of transboundary industrial pollution between two asymmetric nations in infinite-horizon level. In this paper, we model two ways of transboundary pollution: one is an accumulative global pollutant with an uncertain evolutionary dynamic and the other is a regional nonaccumulative pollutant. In our model, firms and governments are separated entities and they play a Stackelberg game, while the governments of the two nations can cooperate in pollution reduction. We discuss the feedback Nash equilibrium strategies of governments and industrial firms, and it is found that the governments being cooperative in transboundary pollution control will set a higher pollution tax rate and make more pollution abatement effort than when they are noncooperative. Additionally, a payment distribution mechanism that supports the subgame consistent solution is proposed.
\end{abstract}

\section{Introduction}

Global environment is a whole ecosystem that is interconnected and indivisible. Any environmental pollution, misuse of resources, and ecological damage risk pressures are likely to be cross-border. Through pathways such as water or air, pollutants can spread across an incredible distance to cause transboundary pollution. Atmospheric pollution and acid rain, ocean and river basin water pollution, hazardous waste transponder movement, and other issues, have been gradually a threat to human survival and development, which become a core issue with international concern. With the development of the industrialization, the transboundary pollution in the world has become increasingly serious. To settle the problem, unilateral response on the part of one nation or region is often ineffective; it calls for cooperation between interested parties $[1,2]$. In this paper, we present a cooperative stochastic differential game model of transboundary industrial pollution between two asymmetric nations. Our objective is to find the optimal pollution abatement strategy which maximizes the social welfare for the two nations gaming for transboundary industrial pollution control.
Differential games have been used as an effective tool to study transboundary pollution control. For example, Dockner and Van Long [3] model a simple dynamic game of two neighboring countries which game for transboundary pollution control; it is found that when the governments of the two countries are restricted to use of linear strategies, noncooperative behavior may result in overall losses for both countries. In 1998, Zagonari extends the model of Dockner and Van Long to analyze a pollution control game between environmentally concerned countries and consumption-oriented countries. In 1993, a tax/subsidy scheme is presented by Martin et al. to combat the transboundary problem of global climate change. Petrosjan and Zaccour [4] apply the Shapley value to determine a fair distribution of the total cooperative cost incurred by countries in a cooperative game of pollution reduction. Bayramoglu [5] uses a dynamic and strategic framework to analyze the transboundary pollution between Romania and Ukraine, it is found that, among the three different institutional systems such as noncooperative game of countries, uniform emission policy, and constant emission policy, the noncooperative game can provide the highest level of total welfare. Jørgensen and Zaccour [6] take emissions and 
investments in abatement technology as control variables to analyze the problem of cooperative transboundary industrial pollution control; in their study, a coordinated solution that maximizes joint welfare is derived together with a payment distribution mechanism that supports the subgame consistent solution. Bertinelli et al. [7] study the strategic behavior of two countries facing transboundary $\mathrm{CO}_{2}$ pollution under a differential game setting; the author considers that feedback strategy may lead to less social waste than when the countries take open-loop strategy. Taking emission permits trading into account; Li [8] studies a differential game of transboundary industrial pollution between two neighboring countries, and the focus of the author's analysis is to find the two countries' noncooperative and cooperative optimal emission paths. Benchekroun and Martín-Herrán [9] study the impact of foresight in a transboundary pollution game; their study shows that when all countries are myopic, that is, choose the "laisser-faire" policy, their payoffs are smaller than when all countries are farsighted.

Most cooperative environmental games do not distinguish the government from the industrial sector in any nation. Apart from these studies, Yeung and Petrosyan [2] take industries and governments as separated entities and develop a cooperative differential game model of transboundary industrial pollution and the time-consistent solutions are derived. Extending the work of Yeung and Petrosyan [2], Huang et al. [10] develop a model in which there is a Stackelberg game between the industrial firms and their local government while the governments can cooperate in transboundary pollution control, and the feedback Nash equilibrium strategies of governments and industrial firms are given. In this paper, we raise a stochastic differential game model of transboundary industrial pollution between two asymmetric countries. Our work can be viewed as a continuing work of Huang et al. [10] in the context of transboundary industrial pollution control. Compared with the work of Huang et al. [10], there are three significant features in our paper. (i) We present a stochastic differential game model of transboundary industrial pollution in which uncertain pollution stock dynamics are taken into account. It is because that uncertain pollution stock dynamics is frequent $[2,11-13]$; actually many factors like unexpected changes in abatement technologies, the unexpected changes of decay rate of the pollutants, and so forth may incur uncertain pollution stock dynamics. (ii) We extend the model set by Huang et al. [10] from finite-horizon level to infinite-horizon level; this is because, in many situations, the terminal time of the game, $T$, is either very far in the future or unknown to the players. A way to resolve the problem, as suggested by Dockner and Nishimura [14], is to set $T=\infty$, so it makes sense to present a model in infinite-horizon level to analyze transboundary industrial pollution control. In our model, we find that the difference of the horizontal level does play an important effect on the behavior of game players. From the government's point of view, the plan is based on long-term interests, so the planning period for the infinite-horizon level is more reasonable. For example, we find that, in the infinite-horizon level, the optimal tax rate and the pollution abatement effort are relatively stable. While, in finite-horizon level, Huang et al. [10] consider the optimal tax rate and the pollution abatement effort decrease in the late game; it means that a hyperopia government can protect the environment better. (iii) In our model, the asymmetry of participants in game is considered; we assume that the industrial firms in one country have more green and energy-efficient technologies than the others, and we show that the one with technology advantage may set a higher pollution tax and make more pollution abatement effort, no matter what condition it would be in, cooperation or noncooperation.

The paper is organized as follows. In Section 2, the game formulation is provided. In Section 3, we characterize the noncooperative outcomes. Cooperative arrangements and individual rationality are analyzed in Section 4. We illustrate the results of a numerical example in Section 5. Section 6 is the results summarizing the paper.

\section{Game Formulation}

2.1. Government and Domestic Industrial Firm: A Stackelberg Game. Consider a multinational economy, which is comprised of two nations. There are $n$ and $m$ industrial firms in nation 1 and nation 2, respectively. In order to control production pollution, the government (the leader) of each nation imposes a pollution tax on domestic industrial firms, and then the industrial firms (the followers) choose their optimal output (emissions) according to the pollution tax rate. This leads to a Stackelberg equilibrium.

Let $\sum_{i=1}^{n} u_{i}(s)$ and $\sum_{j=1}^{m} v_{j}(s)$ denote the quantity of goods produced by $n$ firms in nation 1 and $m$ firms in nation 2 at time $s$, respectively. Firm $i$ 's (or $j$ 's) revenue function $\phi_{i}\left(\right.$ or $\left.\phi_{j}\right)$ is assumed to be concave and increasing, with the following simple functional form:

$$
\begin{aligned}
& \phi_{i}=\beta_{i 1}\left(u_{i}(s)\right)^{1 / 2}, \\
& \phi_{j}=\beta_{j 2}\left(v_{j}(s)\right)^{1 / 2},
\end{aligned}
$$

where $\beta_{i 1}, \beta_{j 2}>0$ are constants; the instantaneous profits of industrial firm in the two nations can be expressed as:

$$
\begin{aligned}
& \phi_{i}\left(u_{i}(s)\right)-c_{1}(s) u_{i}(s), \\
& \phi_{j}\left(v_{j}(s)\right)-c_{2}(s) v_{j}(s),
\end{aligned}
$$

where $c_{k}(s)$ is the pollution tax rate imposed on industrial firms by government $k(k \in 1,2)$ at time $s$.

Through the first-order condition of (2), the optimal yields for the firms in nation 1 and nation 2 could be obtained as follows:

$$
\begin{aligned}
& u_{i}^{*}(s)=\left(\frac{\beta_{i 1}}{2 c_{1}(s)}\right)^{2}, \\
& v_{j}^{*}(s)=\left(\frac{\beta_{j 2}}{2 c_{2}(s)}\right)^{2} .
\end{aligned}
$$

2.2. Local and Global Environmental Impacts. Transboundary pollution may damage the environment through two 
ways, that is, an accumulative global pollutant and a regional nonaccumulative pollutant. For example, some transboundary pollutants are caused by firms such as passing-by waste in waterways, wind-driven suspended particles in air, unpleasant odour, noise, dust, and heat, which are all nonaccumulative pollutants. We assume that the nonaccumulative pollutant, for an output of $u_{i}(s)$ produced by the firm in nation 1 , would cause a short-term impact (cost) of $\varepsilon_{1}^{1} u_{i}(s)$ and $\varepsilon_{1}^{2} u_{i}(s)$ on nation 1 and nation 2 , respectively. Similarly, an output of $v_{j}(s)$ produced by the firm in nation 2 , would cause a short-term (cost) of $\varepsilon_{2}^{1} v_{j}(s)$ and $\varepsilon_{2}^{2} v_{j}(s)$ on nation 1 and nation 2 , respectively. In addition to the nonaccumulative pollutant, there is an accumulative pollutant, such as green-housegas, CFC, and atmospheric particulates; these pollutants can be maintained in environment for a long time and built up existing pollution stocks to create long-term global environmental impacts $[2,5,10,15,16]$. Let $x(s) \subset R^{+}$denote the level of pollution at time $s$, and the dynamics of pollution stock are governed by the stochastic differential equation

$$
\begin{aligned}
d x(s)= & \sum_{i=1}^{n} \alpha_{i 1} u_{i}(s)+\sum_{j=1}^{m} \alpha_{j 2} v_{j}(s) \\
& -\sum_{k=1}^{2} \gamma_{k}\left(I_{k}(s)(x(s))^{1 / 2}-\delta x(s)\right) d s \\
& +\sigma x(s) d z(s), \quad k \in 1,2,
\end{aligned}
$$

where $\sigma$ denotes a noise parameter and $z(s)$ is a Wiener process, $\alpha_{i 1} \geq 0$ and $\alpha_{j 2} \geq 0$ are the amount added to the pollution stock by a unit of firms $i$ 's and $j$ 's output in nation 1 and nation 2 , respectively. $I_{k}(s)$ denotes the pollution abatement effort of nation $k, \gamma_{k}$ is the efficiency of the abatement, $\gamma_{k} I_{k}(s)(x(s))^{1 / 2}$ denotes the amount of pollution removed by $I_{k}(s)$ unit of abatement effort of nation $k$, and $\sigma$ denotes the natural rate of decay of the pollutants.

2.3. The Governments' Objectives. The instantaneous objective of government $k(k \in 1,2)$ at time $s$ can be expressed as

$$
\begin{aligned}
& \sum_{i=1}^{n} \beta_{i 1}\left(u_{i}^{*}(s)\right)^{1 / 2}-\frac{1}{2} a_{1}\left(I_{1}(s)\right)^{2}-\varepsilon_{1}^{1} \sum_{i=1}^{n} u_{i}^{*}(s) \\
& \quad-\varepsilon_{2}^{1} \sum_{j=1}^{m} v_{j}^{*}(s)-h_{1} x(s), \\
& \sum_{j=1}^{m} \beta_{j 1}\left(v_{j}^{*}(s)\right)^{1 / 2}-\frac{1}{2} a_{2}\left(I_{2}(s)\right)^{2}-\varepsilon_{1}^{2} \sum_{i=1}^{n} u_{i}^{*}(s) \\
& \quad-\varepsilon_{2}^{2} \sum_{j=1}^{m} v_{j}^{*}(s)-h_{2} x(s),
\end{aligned}
$$

where $a_{k}, h_{k}>0(k \in 1,2)$ are constants, $(1 / 2) a_{k}\left(I_{k}(s)\right)^{2}$ denotes the cost of employing $I_{k}(s)$ amount of pollution abatement effort, and $h_{k} x(s)$ denotes the value of damage to nation $k$ from $x(s)$ amount of pollution.
The government's planning horizon is $\left[t_{0}, \infty\right)$. The discount rate is $r$. Each government seeks to maximize the integral of its instantaneous objectives (5a) and (5b) over the planning horizon $\left[t_{0}, \infty\right)$ subjected to pollution dynamics (4), with controls on the level of abatement effort and pollution tax.

Substituting $u_{i}(s)$ and $v_{j}(s)$ from (3) into (4) and (5a) and (5b), one obtains a stochastic differential game in which government $k \in 1,2$ seeks

$$
\begin{gathered}
\max _{c_{1}(s), I_{1}(s)} E_{t_{0}} \int_{t_{0}}^{\infty} e^{-r t}\left(\frac{\sum_{i=1}^{n}\left(\beta_{i 1}\right)^{2}}{2 c_{1}(s)}-\frac{1}{2} a_{1}\left(I_{1}(s)\right)^{2}\right. \\
-\frac{\varepsilon_{1}^{1} \sum_{i=1}^{n}\left(\beta_{i 1}\right)^{2}}{4 c_{1}^{2}(s)}-\frac{\varepsilon_{2}^{1} \sum_{j=1}^{m}\left(\beta_{j 2}\right)^{2}}{4 c_{2}^{2}(s)} \\
\left.-h_{1} x(s)\right) d s, \\
\max _{c_{2}(s), I_{2}(s)} E_{t_{0}} \int_{t_{0}}^{\infty} e^{-r t}\left(\frac{\sum_{j=1}^{m}\left(\beta_{j 2}\right)^{2}}{2 c_{2}(s)}-\frac{1}{2} a_{2}\left(I_{2}(s)\right)^{2}\right. \\
\left.-\frac{\varepsilon_{1}^{2} \sum_{i=1}^{n}\left(\beta_{i 1}\right)^{2}}{4 c_{1}^{2}(s)}-\frac{\varepsilon_{2}^{2} \sum_{j=1}^{m}\left(\beta_{j 2}\right)^{2}}{4 c_{2}^{2}(s)}-h_{2} x(s)\right)^{2} d s
\end{gathered}
$$

subject to

$$
\begin{aligned}
d x(s)= & \sum_{i=1}^{n} \alpha_{i 1}\left(\frac{\beta_{i 1}}{2 c_{1}(s)}\right)^{2}+\sum_{j=1}^{m} \alpha_{j 2}\left(\frac{\beta_{j 2}}{2 c_{2}(s)}\right)^{2} \\
& -\sum_{k=1}^{2} \gamma_{k}\left(I_{k}(s)(x(s))^{1 / 2}-\delta x(s)\right) d s \\
& +\sigma x(s) d z(s) .
\end{aligned}
$$

Since the payoffs of nations are measured in monetary terms, the games (6a) and (6b) are a transferable payoff game.

\section{Noncooperative Outcomes}

In this section, the solution of the noncooperative games (6a) and (6b) and (7) will be discussed under a noncooperative framework.

A feedback Nash equilibrium solution can be characterized as follows.

Definition 1. A set of feedback strategies $\left[c_{k}^{*}(t), I_{k}^{*}(t)\right]=$ $\left[\phi_{k}^{c}(t, x), \phi_{k}^{I}(t, x)\right]$, for $k \in 1,2$, provides a Nash equilibrium solution to the games (6a), (6b), and (7), if there exist suitably 
smooth functions $V^{k}(t, x):\left[t_{0}, \infty\right) \times R \rightarrow R, k \in 1,2$, satisfying the following partial differential equations:

$$
\begin{array}{r}
r V^{1}(t, x)-\frac{1}{2} \sigma^{2} x^{2} V_{x x}^{1}(t, x)=\max _{c_{1}(t), I_{1}(t)}\left\{\frac{\sum_{i=1}^{n}\left(\beta_{i 1}\right)^{2}}{2 c_{1}(t)}\right. \\
-\frac{1}{2} a_{1}\left(I_{1}(t)\right)^{2}-\frac{\varepsilon_{1}^{1} \sum_{i=1}^{n}\left(\beta_{i 1}\right)^{2}}{4 c_{1}^{2}(t)}-\frac{\varepsilon_{2}^{1} \sum_{j=1}^{m}\left(\beta_{j 2}\right)^{2}}{4 c_{2}^{2}(t)} \\
-h_{1} x(t)+V_{x}^{1}(t, x)\left(\frac{\sum_{i=1}^{n}\left(\beta_{i 1}\right)^{2}}{4 c_{1}^{2}(t)}+\frac{\sum_{j=1}^{m}\left(\beta_{j 2}\right)^{2}}{4 c_{2}^{2}(t)}\right. \\
\left.\left.-\sum_{k=1}^{2} \gamma_{k}\left(I_{k}(t)(x(t))^{1 / 2}-\delta x(t)\right)\right)\right\}, \\
r V^{2}(t, x)-\frac{1}{2} \sigma^{2} x^{2} V_{x x}^{2}(t, x)=\max _{c_{2}(t), I_{2}(t)}\left\{\frac{\sum_{j=1}^{m}\left(\beta_{j 2}\right)^{2}}{2 c_{2}(t)}\right. \\
-\frac{1}{2} a_{2}\left(I_{2}(t)\right)^{2}-\frac{\varepsilon_{1}^{2} \sum_{i=1}^{n}\left(\beta_{i 1}\right)^{2}}{4 c_{1}^{2}(t)}-\frac{\varepsilon_{2}^{2} \sum_{j=1}^{m}\left(\beta_{j 2}\right)^{2}}{4 c_{2}^{2}(t)} \\
-h_{2} x(t)+V_{x}^{2}(t, x)\left(\frac{\sum_{i=1}^{n}\left(\beta_{i 1}\right)^{2}}{4 c_{1}^{2}(t)}+\frac{\sum_{j=1}^{m}\left(\beta_{j 2}\right)^{2}}{4 c_{2}^{2}(t)}\right. \\
\left.\left.-\sum_{k=1}^{2} \gamma_{k}\left(I_{k}(t)(x(t))^{1 / 2}-\delta x(t)\right)\right)\right\} . \\
\end{array}
$$
yields

Performing the indicated maximization in $(8 \mathrm{a})$ and $(8 \mathrm{~b})$

$$
\begin{aligned}
& \phi_{1}^{c}(t, x)=\varepsilon_{1}^{1}-V_{x}^{1}(t, x), \\
& \phi_{2}^{c}(t, x)=\varepsilon_{2}^{2}-V_{x}^{2}(t, x), \\
& \phi_{1}^{I}(t, x)=-\frac{\gamma_{1} V_{x}^{1}(t, x) x^{1 / 2}(t)}{a_{1}}, \\
& \phi_{2}^{I}(t, x)=-\frac{\gamma_{2} V_{x}^{2}(t, x) x^{1 / 2}(t)}{a_{2}} .
\end{aligned}
$$

Substituting the results of (9a) and (9b) into (8a) and (8b), we obtain the following.

Proposition 2. The government $k$ 's payoff during $\left[t_{0}, \infty\right)$, $V^{k}(t, x)$, can be obtained as

$$
V_{k}(t, x)=\left[A_{k} x(t)+B_{k}\right], \quad \text { for } k \in 1,2,
$$

where $A_{k}, B_{k}$ satisfy the following set of constant coefficient quadratic ordinary differential equations:

$$
\begin{aligned}
& \frac{\gamma_{1}^{2} A_{1}^{2}}{2 a_{1}}+\frac{\gamma_{2}^{2} A_{1} A_{2}}{a_{2}}-\gamma A_{1}-\delta A_{1}-h_{1}=0, \\
& \frac{\gamma_{2}^{2} A_{2}^{2}}{2 a_{2}}+\frac{\gamma_{1}^{2} A_{1} A_{2}}{a_{1}}-\gamma A_{2}-\delta A_{2}-h_{2}=0, \\
& B_{1}=\frac{1}{r}\left(\frac{\sum_{i=1}^{n}\left(\beta_{i 1}\right)^{2}}{4\left(\varepsilon_{1}^{1}-A_{1}\right)}+\frac{\left(A_{1}-\varepsilon_{2}^{1}\right) \sum_{j=1}^{m}\left(\beta_{j 2}\right)^{2}}{4\left(\varepsilon_{2}^{2}-A_{2}\right)^{2}}\right), \\
& B_{2}=\frac{1}{r}\left(\frac{\sum_{j=1}^{m}\left(\beta_{j 1}\right)^{2}}{4\left(\varepsilon_{2}^{2}-A_{2}\right)}+\frac{\left(A_{2}-\varepsilon_{1}^{2}\right) \sum_{i=1}^{n}\left(\beta_{i 1}\right)^{2}}{4\left(\varepsilon_{1}^{1}-A_{1}\right)^{2}}\right) .
\end{aligned}
$$

The corresponding feedback Nash equilibrium of the games (8a) and (8b) can be obtained as

$$
\begin{aligned}
& \phi_{1}^{c}(t, x)=\varepsilon_{1}^{1}-A_{1}, \\
& \phi_{1}^{I}(t, x)=-\frac{\gamma_{1} A_{1} x^{1 / 2}(t)}{a_{1}}, \\
& \phi_{2}^{c}(t, x)=\varepsilon_{2}^{2}-A_{2}, \\
& \phi_{2}^{I}(t, x)=-\frac{\gamma_{2} A_{2} x^{1 / 2}(t)}{a_{2}} .
\end{aligned}
$$

Equation (12) indicates that, under noncooperation, optimal pollution tax rate $\left(\phi_{1}^{c}\right.$ or $\phi_{2}^{c}$ ) may depend on the regional pollutant $\left(\varepsilon_{1}^{1}\right.$ or $\left.\varepsilon_{2}^{2}\right)$ which is caused by the firms in home, but the regional pollutant $\left(\varepsilon_{1}^{2}\right.$ or $\left.\varepsilon_{2}^{1}\right)$ which is caused by the firms in neighbor may have no effect on optimal pollution tax rate. In addition, the optimal abatement effort of the region does not depend on the damage from the regional pollutant at home $\left(\varepsilon_{1}^{1}\right.$ or $\left.\varepsilon_{2}^{2}\right)$; this is because the abatement effort of the nation can only affect pollution stock and cannot affect current pollution.

\section{Cooperative Arrangements}

Now consider the case when both nations cooperate in pollution control. To uphold the cooperative scheme, both group rationality and individual rationality are required to be satisfied at any time.

4.1. Group Optimality and Cooperative State Trajectory. To secure group optimality the participating two nations would 
seek to maximize their joint expected payoff by solving the following stochastic control problem:

$$
\begin{gathered}
W(s, x)=E_{t_{0}} \int_{0}^{\infty}\left\{\frac{\sum_{i=1}^{n}\left(\beta_{i 1}\right)^{2}}{2 c_{1}(s)}+\frac{\sum_{j=1}^{m}\left(\beta_{j 2}\right)^{2}}{2 c_{2}(s)}\right. \\
-\frac{1}{2} \sum_{k=1}^{2} a_{k}\left(I_{k}(s)\right)^{2}-\frac{\left(\varepsilon_{1}^{1}+\varepsilon_{1}^{2}\right) \sum_{i=1}^{n}\left(\beta_{i 1}\right)^{2}}{4 c_{1}^{2}(s)} \\
\left.-\frac{\left(\varepsilon_{2}^{1}+\varepsilon_{2}^{2}\right) \sum_{j=1}^{m}\left(\beta_{j 2}\right)^{2}}{4 c_{2}^{2}(s)}-\left(h_{1}+h_{2}\right) x(s)\right\} e^{-r t} d t
\end{gathered}
$$

subject to (7).

Invoking Bellman's technique, a set of controls $\left\{\widehat{c}_{k}^{*}, \widehat{I}_{k}^{*}\right\}=$ $\left\{\widehat{\phi}_{k}^{c}(x), \widehat{\phi}_{k}^{I}(x)\right\}$ constitutes an optimal solution to the stochastic control problem (13) and (7), if there exists continuously differentiable function $W(t, x):[t, \infty) \times R \rightarrow R, k \in 1,2$, satisfying the following partial differential equations:

$$
\begin{aligned}
& r W(t, x)-\frac{1}{2} \sigma^{2} x^{2} W_{x x}(t, x)=\max _{c_{1}, I_{1}, c_{2}, I_{2}}\left\{\frac{\sum_{i=1}^{n}\left(\beta_{i 1}\right)^{2}}{2 c_{1}(t)}\right. \\
& +\frac{\sum_{j=1}^{m}\left(\beta_{j 2}\right)^{2}}{2 c_{2}(t)}-\frac{1}{2} \sum_{k=1}^{2} a_{k}\left(I_{k}(t)\right)^{2} \\
& -\frac{\left(\varepsilon_{1}^{1}+\varepsilon_{1}^{2}\right) \sum_{i=1}^{n}\left(\beta_{i 1}\right)^{2}}{4 c_{1}^{2}(t)}-\frac{\left(\varepsilon_{2}^{1}+\varepsilon_{2}^{2}\right) \sum_{j=1}^{m}\left(\beta_{j 2}\right)^{2}}{4 c_{2}^{2}(t)} \\
& -\left(h_{1}+h_{2}\right) x(t)+W_{X}(t, x)\left(\frac{\sum_{i=1}^{n} \alpha_{i 1}\left(\beta_{i 1}\right)^{2}}{4 c_{1}^{2}(t)}\right. \\
& +\frac{\sum_{j=1}^{m} \alpha_{j 2}\left(\beta_{j 2}\right)^{2}}{4 c_{2}^{2}(t)} \\
& \left.\left.+\sum_{k=1}^{2} \gamma_{k}\left(I_{k}(t)(x(t))^{1 / 2}-\delta x(t)\right)\right)\right\}
\end{aligned}
$$

Performing the indicated maximization in (14) yields the optimal controls under cooperation:

$$
\begin{aligned}
\widehat{\phi}_{1}^{c}(t, x) & =\varepsilon_{1}^{1}+\varepsilon_{1}^{2}-W_{x}(t, x), \\
\widehat{\phi}_{2}^{c}(t, x) & =\varepsilon_{2}^{1}+\varepsilon_{2}^{2}-W_{x}(t, x), \\
\widehat{\phi}_{1}^{I}(x) & =-\frac{\gamma_{1} W_{x}(x) x^{1 / 2}}{a_{1}}, \\
\widehat{\phi}_{2}^{I}(x) & =-\frac{\gamma_{2} W_{x}(x) x^{1 / 2}}{a_{2}} .
\end{aligned}
$$

Substituting the results in (15a) and (15b) into (14), we obtain the following.
Proposition 3. System (13) admits a solution

$$
W(t, x)=[\widehat{A} x(t)+\widehat{B}],
$$

where $\widehat{A}, \widehat{B}$ satisfy the following set of constant coefficient quadratic ordinary differential equations:

$$
\begin{aligned}
& \left(\frac{\gamma_{1}^{2}}{2 a_{1}}+\frac{\gamma_{2}^{2}}{2 a_{2}}\right) \widehat{A}^{2}-(r+\delta) \widehat{A}-\left(h_{1}+h_{2}\right)=0, \\
& \widehat{B}=\frac{1}{r}\left(\frac{\sum_{i=1}^{n}\left(\beta_{i 1}\right)^{2}+\sum_{j=1}^{m}\left(\beta_{j 2}\right)^{2}}{4\left(\varepsilon_{1}^{1}+\varepsilon_{2}^{2}-\widehat{A}\right)^{2}}\right) .
\end{aligned}
$$

The corresponding feedback Nash equilibriums under cooperation can be obtained as

$$
\begin{aligned}
& \widehat{\phi}_{1}^{c}(t, x)=\varepsilon_{1}^{1}+\varepsilon_{2}^{2}-\widehat{A}, \\
& \widehat{\phi}_{1}^{I}(t, x)=-\frac{\gamma_{1} \widehat{A} x^{1 / 2}(t)}{2 a_{1}}, \\
& \widehat{\phi}_{2}^{c}(t, x)=\varepsilon_{1}^{1}+\varepsilon_{2}^{2}-\widehat{A}, \\
& \widehat{\phi}_{2}^{I}(t, x)=-\frac{\gamma_{2} \widehat{A} x^{1 / 2}(t)}{2 a_{2}} .
\end{aligned}
$$

Substituting the optimal control strategy from (18) into (7) yields the dynamics of pollution accumulation under cooperation:

$$
\begin{aligned}
& d x(s)=\left(\frac{\sum_{i=1}^{n} \alpha_{i 1}\left(\beta_{i 1}\right)^{2}+\sum_{j=1}^{m} \alpha_{j 2}\left(\beta_{j 2}\right)^{2}}{4\left(\varepsilon_{1}^{1}+\varepsilon_{2}^{2}-\widehat{A}\right)^{2}}\right. \\
& \left.\quad+\sum_{k=1}^{2} \frac{\left(\gamma_{k}\right)^{2} \widehat{A} x(s)}{2 a_{k}}-\delta x(s)\right) d s+\sigma x(s) d z(s), \\
& x\left(t_{0}\right)=x_{0} .
\end{aligned}
$$

Solving the stochastic cooperative pollution dynamics yields the cooperative state trajectory:

$$
\begin{gathered}
x^{*}(t)=e^{\left[\int_{t_{0}}^{t}\left[\left(\gamma_{1}^{2} / 2 a_{1}+\gamma_{2}^{2} / 2 a_{2}\right) \widehat{A}-\delta-\sigma / 2\right] d s+\int_{t_{0}}^{t} \sigma d z(s)\right]} \times\left[x_{t_{0}}\right. \\
+\int_{t_{0}}^{t}\left(\frac{\sum_{i=1}^{n} \alpha_{i 1}\left(\beta_{i 1}\right)^{2}+\sum_{j=1}^{m} \alpha_{j 2}\left(\beta_{j 2}\right)^{2}}{4\left(\varepsilon_{1}^{1}+\varepsilon_{2}^{2}-\widehat{A}\right)^{2}}\right) \\
\left.\times e^{\left[\int_{t_{0}}^{t}\left[\sigma^{2} / 2+\delta-\left(\gamma_{1}^{2} / 2 a_{1}+\gamma_{2}^{2} / 2 a_{2}\right) \widehat{A}\right] d \tau+\int_{t_{0}}^{t} \sigma d z(s)\right]} d s\right], \\
\text { for } t \in\left[t_{0}, \infty\right) .
\end{gathered}
$$

4.2. Individually Rational and Time-Consistent Imputation and Payment Distribution Mechanism. Let $X_{t}^{*}$ denote the 
set of realizable values of $x^{*}(t)$ at time $t$ generated by (20). The term $x_{t}^{*}$ is used to denote an element of the set $X_{t}^{*}$. An agreed upon optimality principle would be sought to allocate the cooperative payoff. In a dynamic framework, individual rationality has to be maintained at every instant of time within the cooperative duration $\left[t_{0}, \infty\right)$ along the cooperative trajectory (20). For $\tau \in\left[t_{0}, \infty\right)$, let vector $\xi^{k}\left(x_{t}^{*}\right)=\left\{\xi^{1}\left(x_{t}^{*}\right), \xi^{2}\left(x_{t}^{*}\right)\right\}$ denote the solution imputation (payoff under cooperation) over the period $[\tau, \infty)$ to player $k \in\{1,2\}$ given that the state is $x_{\tau}^{*} \in X_{\tau}^{*}$. Individual rationality along the cooperative trajectory requires

$$
\xi^{(\tau) k}\left(\tau, x_{t}^{*}\right) \geq V^{(\tau) k}\left(\tau, x_{t}^{*}\right), \quad \text { for } k \in\{1,2\}
$$

where $V^{(\tau) k}\left(\tau, x_{t}^{*}\right)$ denotes the payoff to nation $k$ under noncooperation over the period. Let $G(s)=\left[G_{1}(s), G_{2}(s)\right]$ denote the instantaneous payoff of the cooperative game at time $s \in\left[t_{0}, \infty\right)$ for the cooperative $\Gamma_{c}\left(x_{t_{0}}^{*}\right)$.

Theorem 4. An instantaneous payment at time $\tau \in\left[t_{0}, T\right]$ equaling

$$
\begin{aligned}
& G_{K}(\tau)=r \xi^{k}\left(x_{\tau}^{*}\right)-\frac{1}{2} \sigma^{2}\left(x_{\tau}^{*}\right)^{2} \xi_{x_{\tau}^{*} x_{\tau}^{*}}^{k}\left(x_{\tau}^{*}\right)-\xi_{x_{\tau}^{*}}^{k}\left(x_{\tau}^{*}\right) \\
& \cdot\left(\sum_{i=1}^{n} \alpha_{i 1}\left(\frac{\beta_{i 1}}{2 c_{1}\left(\tau, x_{\tau}^{*}\right)}\right)^{2}+\sum_{j=1}^{m} \alpha_{j 2}\left(\frac{\beta_{j 2}}{2 c_{2}\left(\tau, x_{\tau}^{*}\right)}\right)^{2}\right. \\
& \left.-\sum_{k=1}^{2} \gamma_{k} I_{k}\left(\tau, x_{\tau}^{*}\right)\left(x_{\tau}^{*}\right)^{1 / 2}-\delta x_{\tau}^{*}\right), \\
& \text { for } \tau \in\left[t_{0}, \infty\right), x_{\tau}^{*} \in X_{\tau}^{*}, k \in\{1,2\}
\end{aligned}
$$

yields a subgame consistent solution to the cooperative game $\Gamma_{c}\left(x_{t_{0}}^{*}\right)$.

Proof. Along the cooperative trajectory $\left\{x^{*}(t)\right\}_{t \geq t_{0}}$, we have

$$
\begin{aligned}
& \Upsilon^{k}\left(\tau, \tau, x_{\tau}^{*}\right)=\xi^{k}\left(x_{\tau}^{*}\right) \\
& \quad=E_{\tau}\left\{\int_{\tau}^{\infty} G_{k}(s) e^{-r(s-\tau)} d s \mid x(\tau)=x_{\tau}^{*}\right\}, \\
& \Upsilon^{k}\left(\tau, t, x_{t}^{*}\right)=\xi^{k}\left(x_{t}^{*}\right) \\
& \quad=E_{t}\left\{\int_{t}^{\infty} G_{k}(s) e^{-r(s-t)} d s \mid x(t)=x_{t}^{*}\right\}, \\
& \quad \text { for } k \in\{1,2\}, x_{t}^{*} \in X_{t}^{*}, t \geq \tau .
\end{aligned}
$$

Note that

$$
\begin{array}{r}
\Upsilon^{k}\left(\tau ; t, x_{t}^{*}\right)=e^{-r(s-\tau)} \xi\left(x_{t}^{*}\right)=e^{-r(s-\tau)} \Upsilon^{k}\left(t ; t, x_{t}^{*}\right), \\
k \in\{1,2\}, x_{\tau}^{*} \in X_{\tau}^{*} .
\end{array}
$$

Since $\Upsilon^{k}\left(t ; t, x_{t}^{*}\right)$ is continuously differentiable in $t$ and $x_{t}^{*}$, one can obtain

$$
\begin{aligned}
& \Upsilon^{k}\left(\tau, \tau, x_{\tau}^{*}\right)=E_{\tau}\left\{\int_{\tau}^{\tau+\Delta t} G_{k}(s) e^{-r(s-\tau)} d s\right. \\
& \quad+e^{-r(\Delta t)} \Upsilon^{k}\left(\tau+\Delta t ; \tau+\Delta t, x_{\tau}^{*}+\Delta x_{\tau}^{*}\right) \mid x(\tau) \\
& \left.\quad=x_{\tau}^{*}\right\}, \quad \text { for } \tau \in\left[t_{0}, \infty\right), x_{\tau}^{*} \in X_{\tau}^{*}, k \in\{1,2\},
\end{aligned}
$$

where

$$
\begin{aligned}
& \Delta x_{\tau}^{*} \\
& =\sum_{i=1}^{n} \alpha_{i 1}\left(\frac{\beta_{i 1}}{2 c_{1}\left(\tau, x_{\tau}^{*}\right)}\right)^{2}+\sum_{j=1}^{m} \alpha_{j 2}\left(\frac{\beta_{j 2}}{2 c_{2}\left(\tau, x_{\tau}^{*}\right)}\right)^{2} \\
& -\sum_{k=1}^{2} \gamma_{k}\left(I_{K}\left(\tau, x_{\tau}^{*}\right)\left(x_{\tau}^{*}\right)^{1 / 2}-\delta x_{\tau}^{*}\right) \Delta t \\
& +\sigma\left[x_{\tau}^{*}\right] \Delta z_{\tau}+o(\Delta t), \\
& \Delta z_{\tau}=z(\tau+\Delta t)-z(\tau), \\
& \frac{E_{\tau}[o(\Delta t)]}{\Delta t} \longrightarrow 0,
\end{aligned}
$$

From (24) and (25), one can obtain

$$
\begin{aligned}
\Upsilon^{k}\left(\tau, \tau, x_{\tau}^{*}\right)= & E_{\tau}\left\{\int_{\tau}^{\tau+\Delta t} G_{k}(s) e^{-r(s-\tau)} d s\right. \\
\left.+\Upsilon^{k}\left(\tau ; \tau+\Delta t, x_{\tau}^{*}+\Delta x_{\tau}^{*}\right) \mid x(\tau)=x_{\tau}^{*}\right\}, & \\
& \text { for } \tau \in\left[t_{0}, \infty\right), x_{\tau}^{*} \in X_{\tau}^{*}, k \in\{1,2\} .
\end{aligned}
$$

Therefore, with $\Delta t \rightarrow 0,(27)$ can be expressed as

$$
\begin{gathered}
G_{k}(\tau) \Delta t=-\left[\left.\Upsilon_{t}^{k}\left(\tau ; t, x_{\tau}^{*}\right)\right|_{t=\tau}\right] \Delta t-\frac{1}{2} \sigma^{2}\left(x_{\tau}^{*}\right)^{2} \\
\cdot\left[\left.\Upsilon_{x_{\tau}^{*} x_{\tau}^{*}}^{k}\left(\tau ; t, x_{\tau}^{*}\right)\right|_{t=\tau}\right] \Delta t-\left[\left.\Upsilon_{x_{\tau}^{*}}^{k}\left(\tau ; t, x_{\tau}^{*}\right)\right|_{t=\tau}\right] \\
\times\left(\sum_{i=1}^{n} \alpha_{i 1}\left(\frac{\beta_{i 1}}{2 c_{1}\left(\tau, x_{\tau}^{*}\right)}\right)^{2}+\sum_{j=1}^{m} \alpha_{j 2}\left(\frac{\beta_{j 2}}{2 c_{2}\left(\tau, x_{\tau}^{*}\right)}\right)^{2}\right. \\
\left.\quad-\sum_{k=1}^{2} \gamma_{k}\left(I_{K}\left(\tau, x_{\tau}^{*}\right)\left(x_{\tau}^{*}\right)^{1 / 2}-\delta x_{\tau}^{*}\right) \Delta t-o(\Delta t)\right) .
\end{gathered}
$$

Dividing (28) throughout by $\Delta t$, with $\Delta t \rightarrow 0$, and taking expectation, one can obtain (22). Hence Theorem 4 follows.

When the two nations adopt the cooperative strategies, the rate of instantaneous payment that nation $k(k \in\{1,2\})$ 
TABLE 1: The parameters of model used in the numerical solutions.

\begin{tabular}{lcccccccccccc}
\hline$t_{0}$ & $\beta_{11}$ & $\beta_{21}$ & $\beta_{12}$ & $\beta_{22}$ & $x\left(t_{0}\right)$ & $\alpha_{11}$ & $\alpha_{21}$ & $\alpha_{12}$ & $\alpha_{22}$ & $\gamma_{1}$ \\
\hline 0 & 60 & 90 & 40 & 70 & 20 & 0.4 & 0.3 & 0.5 & 0.4 & 0.4 & 0.2 \\
$\delta$ & $\sigma$ & $a_{1}$ & $a_{2}$ & $\varepsilon_{1}^{1}$ & $\varepsilon_{1}^{2}$ & $\varepsilon_{2}^{2}$ & $\varepsilon_{2}^{1}$ & $r$ & $h_{1}$ & $h_{2}$ & 5 \\
0.01 & 0.05 & 0.8 & 1 & 0.2 & 0.15 & 0.15 & 0.16 & 0.05 & 4 & 5 \\
\hline
\end{tabular}

will realize at time $\tau$ with the state being $x_{\tau}^{*}$ can be expressed as

$$
\begin{aligned}
& S_{1}(\tau, x)=\frac{r}{2}\left\{\left[A_{1} x_{\tau}^{*}+B_{1}\right]+\left[\widehat{A} x_{\tau}^{*}+\widehat{B}\right]\right. \\
& \left.-\left[A_{2} x_{\tau}^{*}+B_{2}\right]\right\}-\frac{1}{2}\left[A_{1}+\widehat{A}-A_{2}\right] \\
& \cdot\left\{\frac{\sum_{i=1}^{n}\left(\beta_{i 1}\right)^{2}+\sum_{j=1}^{m}\left(\beta_{j 2}\right)^{2}}{4\left(\varepsilon_{1}^{1}+\varepsilon_{2}^{2}-\widehat{A}\right)^{2}}\right. \\
& \left.+\sum_{k=1}^{2} \frac{\gamma_{k}^{2} \widehat{A} x_{\tau}^{*}}{2 a_{k}}-\delta x_{\tau}^{*}\right\}, \\
& S_{2}(\tau, x)=\frac{r}{2}\left\{\left[A_{2} x_{\tau}^{*}+B_{2}\right]+\left[\widehat{A} x_{\tau}^{*}+\widehat{B}\right]\right. \\
& \left.\quad-\left[A_{1} x_{\tau}^{*}+B_{1}\right]\right\}-\frac{1}{2}\left[A_{2}+\widehat{A}-A_{1}\right] \\
& +\left\{\frac{\sum_{i=1}^{n}\left(\beta_{i 1}\right)^{2}+\sum_{j=1}^{m}\left(\beta_{j 2}\right)^{2}}{4\left(\varepsilon_{1}^{1}+\varepsilon_{2}^{2}-\widehat{A}\right)^{2}}\right. \\
& \left.+\sum_{k=1}^{2} \frac{\gamma_{k}^{2} \widehat{A} x_{\tau}^{*}}{2 a_{k}}-\delta x_{\tau}^{*}\right\} .
\end{aligned}
$$

\section{Numerical Example}

Consider a multinational economy consisting of two nations, and each with two industrial firms. Industrial firm's revenue function in nation 1 and nation 2 at time $s$ is $\phi_{i}=\beta_{i 1}\left(u_{i}(s)\right)^{1 / 2}$ and $\phi_{j}=\beta_{j 2}\left(v_{j}(s)\right)^{1 / 2}(i, j=1,2)$, respectively, where $\beta_{11}=60, \beta_{21}=90, \beta_{12}=40$, and $\beta_{22}=70$. The value of $\beta_{i 1}(i \in\{1,2\})$ is higher than $\beta_{j 2}(j \in\{1,2\})$; it means that, compared with industrial firms in nation 2 , industrial firms in nation 1 have more energy-efficient technology. In addition, industrial firms in nation 1 have technical advantage result in $\alpha_{11}<\alpha_{12}, \alpha_{21}<\alpha_{22}, \gamma_{1}>\gamma_{2}$. As mentioned above, the optimal pollution emissions of the firms in nations 1 and 2 are $u_{i}^{*}(s)=\left(\beta_{i 1} / 2 c_{1}(s)\right)^{2}$ and $v_{j}^{*}(s)=\left(\beta_{j 2} / 2 c_{2}(s)\right)^{2}$, respectively. The dynamics of pollution stock are governed by the stochastic differential equation (4), where $x\left(t_{0}\right)=20$, $\delta=0.01$, and $\sigma=0.05$. We defined $a_{k}$ as the amount added to the pollution stock by a unit of output in nation $k(k=1,2)$. They are a positive number less than 1 ; that is to say, the stock of pollution will increase with the output but not higher than the amount of output. According to
TABLE 2: The optimal pollution tax rate.

\begin{tabular}{lccc}
\hline$c_{1}(t)$ & $c_{2}(t)$ & $\widehat{c}_{1}(t)$ & $\widehat{c}_{2}(t)$ \\
\hline 8.20 & 2.34 & 10.90 & 10.87 \\
\hline
\end{tabular}

experience, we assign $\alpha_{11}=0.4, \alpha_{21}=0.3, \alpha_{12}=0.5$, $\alpha_{22}=0.4 \cdot \gamma_{k}(k=1,2)$ can also be considered as pollution abatement effort efficiency, which is linked to pollution control policies and environmental governance efficiency in different nations. Generally speaking, pollution abatement effort efficiency is higher in developed countries. We assign $\gamma_{1}=0.4, \gamma_{2}=0.2$. Abatement costs are $0.4\left(I_{1}(s)\right)^{1 / 2}$ and $0.5\left(I_{2}(s)\right)^{1 / 2}$ for nations 1 and 2 , respectively. Local pollution impacts are closely linked to the level of production activities. The accumulation of pollution stock like greenhouse gas often concerns the interactions between the natural environment and the pollutants emitted. So stochastic elements would appear. According to what we defined before, $\varepsilon_{k}^{1}, \varepsilon_{k}^{2}(k=1,2)$ is a pollution damage cost coefficients. It is said that the bigger they are, the higher the cost of environmental damage for this nation is. We make the following assignments: $\varepsilon_{1}^{1}=0.2$, $\varepsilon_{1}^{2}=0.15, \varepsilon_{2}^{2}=0.15$, and $\varepsilon_{2}^{1}=0.16 . h_{k}(k=1,2)$ are the value of pollution damage to nation $k(k=1,2)$. The larger they are, the greater the impact of the environment is, which is determined by the different regions. And we make assignments: $h_{1}=4, h_{2}=5$. Other parameters are as follows: $r=0.05, t_{0}=0$. The parameters of the model, which are used in the numerical solution, are presented in Table 1.

Then we obtain a stochastic differential game in which government 1 and government 2 under noncooperation seek

$$
\begin{aligned}
& \max _{c_{1}(s), I_{1}(s)} \int_{0}^{\infty} e^{-r t}\left(\frac{5850}{c_{1}(s)}-0.4 I_{1}^{2}(s)-\frac{585}{c_{1}^{2}(s)}-\frac{162.2}{c_{2}^{2}(s)}\right. \\
& \quad-4 x(s)) d x \\
& \max _{c_{2}(s), I_{2}(s)} \int_{0}^{\infty} e^{-r t}\left(\frac{3250}{c_{2}(s)}-0.5 I_{2}^{2}(s)-\frac{438.75}{c_{1}^{2}(s)}\right. \\
& \left.-\frac{243.75}{c_{2}^{2}(s)}-5 x(s)\right) d x .
\end{aligned}
$$

Through the analysis mentioned above, we have obtained the pollution tax rate, abatement effort of each nation, and the dynamics of pollution stock under noncooperative game and cooperative game. Now we will analyze their difference between the governments under noncooperation and cooperation through Table 2 and Figures 1-5. 


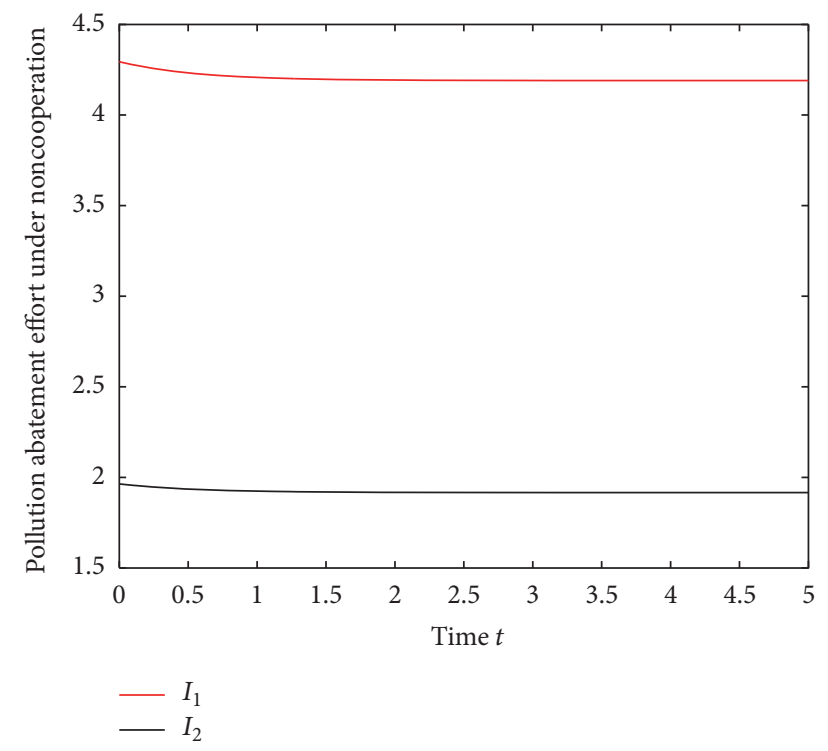

FIGURE 1: The pollution abatement effort under noncooperation, where $I_{1}$ and $I_{2}$ denote the pollution abatement effort of governments under noncooperation in nations 1 and 2 , respectively.

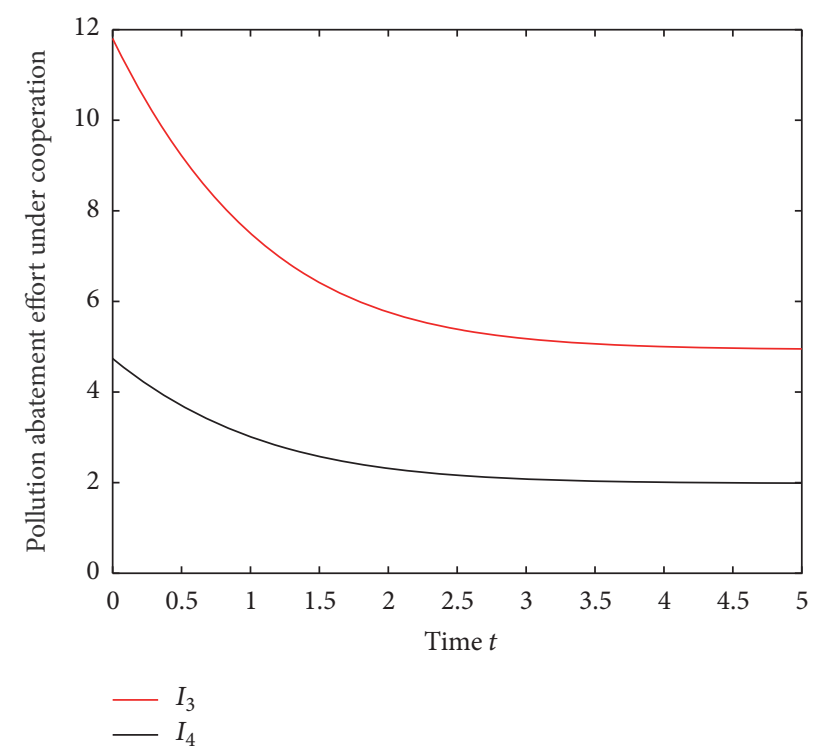

FIgURE 2: The pollution abatement effort under cooperation, where $I_{3}$ and $I_{4}$ are the pollution abatement effort of governments under cooperation in nations 1 and 2 , respectively.

From Table 2, we can see that, for each government, the noncooperative optimal tax rates $c_{1}(t)$ and $c_{2}(t)$ are less than the cooperative tax rates $\widehat{c}_{1}(t)$ and $\widehat{c}_{2}(t)$. This means that cooperation would push to raise the tax rate to reduce production and cut pollution. And, for both governments, the optimal pollution tax rate is not changing with time; this is different from Huang et al. [10] and Yeung and Petrosyan [2], because, in their research, they model a finite-horizon level game regardless of cooperative game or noncooperative game, and, in the later stage of game, both governments would pay more attention to the economic

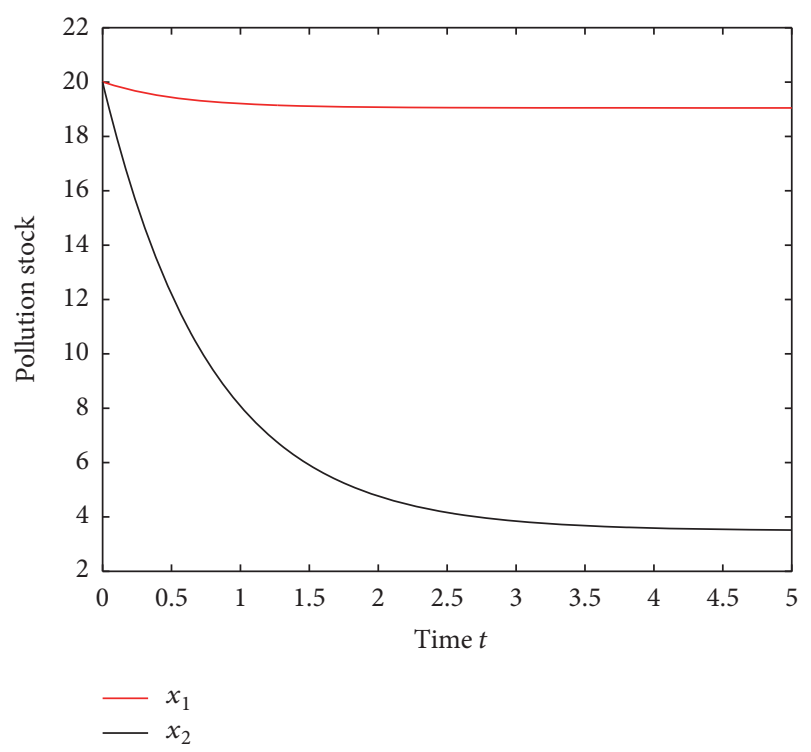

FIGURE 3: The dynamics of pollution stock under noncooperation or cooperation, where $x_{1}$ and $x_{2}$ denote the pollution stock under noncooperation and cooperation, respectively.

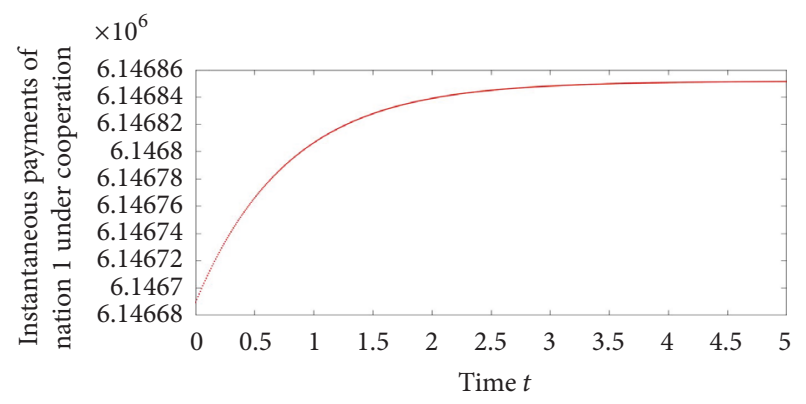

FIGURE 4: The instantaneous payments of nation 1 under cooperation.

benefits than pollution abatement. Then they would decrease the pollution tax rate to encourage production and stimulate economic development [10]. But, in our model, the game is in infinite-horizon, and there is no later stage of the game, so the optimal pollution tax rate will not decrease. In addition, under noncooperation, the governments take the externalities of production into account including their own nation only. However, under a cooperation, the externalities are considered by the governments, which include their own nation and the neighbors, so the optimal pollution tax rate is higher than under noncooperation.

Figures 1 and 2 show the pollution abatement effort of both governments. From them we find that, under noncooperation, the pollution abatement effort of both governments in the two nations could remain stable all the time, but, under cooperation, both governments would make a high pollution abatement effort at first, and then, the effort level may reduce to a certain degree; after that, it would tend to remain stable. For each government, the noncooperative pollution abatement effort is lower than the cooperative pollution abatement effort. This is determined by externality 


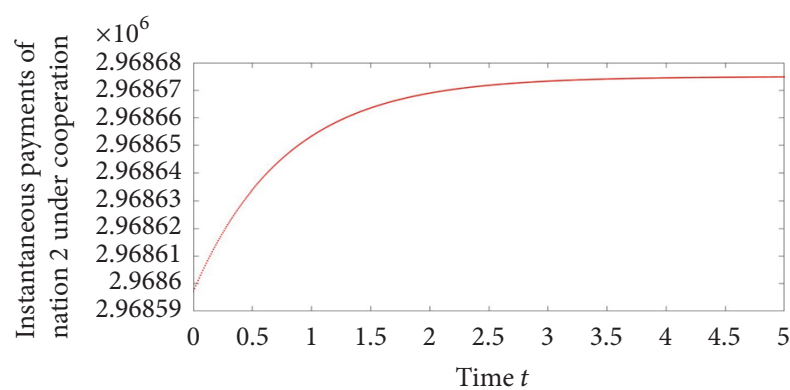

FIGURE 5: The instantaneous payments of nation 2 under cooperation.

of pollution emissions. Under cooperative game, the governments pay attention to the damage for the entire region, so marginal damage of pollution emission would be greater and the governments would be more concerned about pollutant reduction.

Figure 3 shows the dynamics of pollution stock under noncooperation or cooperation. We can see that, the pollution stock under noncooperative game is higher than that under cooperative game. It is due to the higher pollution tax rate and higher pollution abatement effort of both governments under cooperative game. Then, in both economic and environmental terms, cooperation is always better than noncooperation.

Now consider the case when the two nations want to cooperate. To secure group optimality the two nations seek to maximize their joint expected payoff by solving the following stochastic control problem:

$$
\begin{aligned}
& \max _{c_{1}(s), c_{2}(s), I_{1}(s), I_{2}(s)} E_{0} \int_{0}^{\infty}\left(\frac{5850}{c_{1}(s)}+\frac{3250}{c_{2}(s)}-0.4\left(I_{1}(s)\right)^{2}\right. \\
& \left.-0.5\left(I_{2}(s)\right)^{2}-\frac{1023.75}{\left(c_{1}(s)\right)^{2}}-\frac{503.75}{\left(c_{2}(s)\right)^{2}}-10 x(s)\right) \\
& \cdot e^{-0.05} d t
\end{aligned}
$$

subject to

$$
d x(s)=3.49-1.28 x(s)+0.05 x(s) d z(s) .
$$

According to Theorem 4, we get the appropriate dynamic distribution scheme that both group optimality and individual rationality are satisfied at any time during cooperative game. The instantaneous payments of two nations, $S_{1}\left(t, x_{t}^{*}\right)$ and $S_{2}\left(t, x_{t}^{*}\right)$, at different time $t$ with given $x_{t}^{*}$ are shown in (33a) and (33b) and Figures 3 and 4.

$$
\begin{aligned}
S_{1}\left(t, x_{t}^{*}\right)= & \frac{1007100178948148626983247}{163840000000000000} \\
& -\frac{12670021998361 e^{-32 t / 25}}{78125000000},
\end{aligned}
$$

$$
\begin{aligned}
S_{2}\left(t, x_{t}^{*}\right)= & \frac{7782203372235146274391153}{2621440000000000000} \\
& -\frac{1518945509601 e^{-32 t / 25}}{19531250000} .
\end{aligned}
$$

It is shown in Figures 4 and 5 that the instantaneous payment of both nations which satisfy the property of subgame consistency shows an increasing trend at the start and then remains stable. In addition, the instantaneous payment for nation 1 is always higher than that of nation 2 . This is because the industrial firms in nation 1 have more green and energyefficient technology, so they would get more instantaneous payment.

\section{Conclusions}

In this paper, we have shown a stochastic differential game of transboundary industrial pollution between two asymmetric nations in infinite-horizon level. In our model, the firms and governments are separate entities. As participants in the games, they take game behavior to optimize their own payment, and the game equilibrium has been solved under the two levels of firms and governments simultaneously. The uncertain evolution dynamic of pollution stock that has a long-term global impact has been taken into account in our models. Otherwise, another negative environmental externality, a regional nonaccumulative pollutant that causes a short-term local impact, has also been considered. We characterize the parameters of spaces where two neighboring governments can cooperate and give the feedback Nash equilibrium strategies of governments and industrial firms. Through analysis, we have shown that the optimal pollution tax rate hinges on the two effects of regional damage: the source region and the global pollution stock, under noncooperative game, while, under cooperative game, in addition to the above two kinds of effects, it also hinges on the additional effect of regional damage coming from the neighborhood, so the latter is greater than the former. Moreover, we have shown that the pollution abatement effort under cooperation is more than under noncooperation. By analyzing this stochastic differential game model, we have also provided a payment distribution mechanism, which supports the subgame consistent solution, and present a numerical example to illustrate the implementation of this mechanism.

\section{Conflicts of Interest}

The authors declare that there are no conflicts of interest regarding the publication of this paper.

\section{Acknowledgments}

The authors would like to acknowledge the financial support from the China National Funding for Social Science Research Project (Project no. 15CJY037) and the Philosophy and Social Science Foundation of Hunan, China (Project no. 16YBA315), for this paper. 


\section{References}

[1] D. W. Yeung, "Dynamically consistent cooperative solution in a differential game of transboundary industrial pollution," Journal of Optimization Theory and Applications, vol. 134, no. 1, pp. 143-160, 2007.

[2] D. W. Yeung and L. A. Petrosyan, "A cooperative stochastic differential game of transboundary industrial pollution," Automatica, vol. 44, no. 6, pp. 1532-1544, 2008.

[3] E. J. Dockner and N. Van Long, "International pollution control: cooperative versus noncooperative strategies," Journal of Environmental Economics and Management, vol. 25, no. 1, pp. 13-29, 1993.

[4] L. Petrosjan and G. Zaccour, "Time-consistent Shapley value allocation of pollution cost reduction," Journal of Economic Dynamics \& Control, vol. 27, no. 3, pp. 381-398, 2003.

[5] B. Bayramoglu, "Transboundary pollution in the black sea: comparison of institutional arrangements," Environmental and Resource Economics, vol. 35, no. 4, pp. 289-325, 2006.

[6] S. Jørgensen and G. Zaccour, "Incentive equilibrium strategies and welfare allocation in a dynamic game of pollution control," Automatica, vol. 37, no. 1, pp. 29-36, 2001.

[7] L. Bertinelli, C. Camacho, and B. Zou, "Carbon capture and storage and transboundary pollution: a differential game approach," European Journal of Operational Research, vol. 237, no. 2, pp. 721-728, 2014.

[8] S. Li, "A differential game of transboundary industrial pollution with emission permits trading," Journal of Optimization Theory and Applications, vol. 163, no. 2, pp. 642-659, 2014.

[9] H. Benchekroun and G. Martín-Herrán, "The impact of foresight in a transboundary pollution game," European Journal of Operational Research, vol. 251, no. 1, pp. 300-309, 2016.

[10] X. Huang, P. He, and W. Zhang, "A cooperative differential game of transboundary industrial pollution between two regions," Journal of Cleaner Production, vol. 120, pp. 43-52, 2016.

[11] A. Haurie, J. B. Krawczyk, and M. Roche, "Monitoring cooperative equilibria in a stochastic differential game," Journal of Optimization Theory and Applications, vol. 81, no. 1, pp. 73-95, 1994.

[12] E. Saltari and G. Travaglini, "The effects of environmental policies on the abatement investment decisions of a green firm," Resource and Energy Economics, vol. 33, no. 3, pp. 666-685, 2011.

[13] S. Athanassoglou and A. Xepapadeas, "Pollution control with uncertain stock dynamics: when, and how, to be precautious," Journal of Environmental Economics and Management, vol. 63, no. 3, pp. 304-320, 2012.

[14] E. J. Dockner and K. Nishimura, "Transboundary pollution in a dynamic game model," Japanese Economic Review, vol. 50, no. 4, pp. 443-456, 1999.

[15] F. Bosello, B. Buchner, and C. Carraro, "Equity, development, and climate change control," Journal of the European Economic Association, vol. 1, no. 2-3, pp. 601-611, 2003.

[16] A. Bernard, A. Haurie, M. Vielle, and L. Viguier, "A two-level dynamic game of carbon emission trading between Russia, China, and annex B countries," Journal of Economic Dynamics and Control, vol. 32, no. 6, pp. 1830-1856, 2008. 


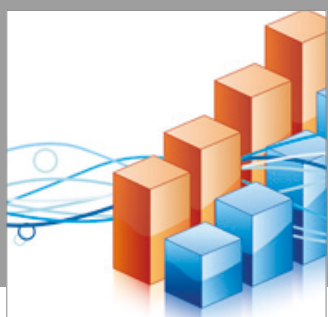

Advances in

Operations Research

vatersals

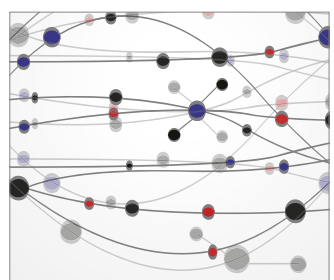

\section{The Scientific} World Journal
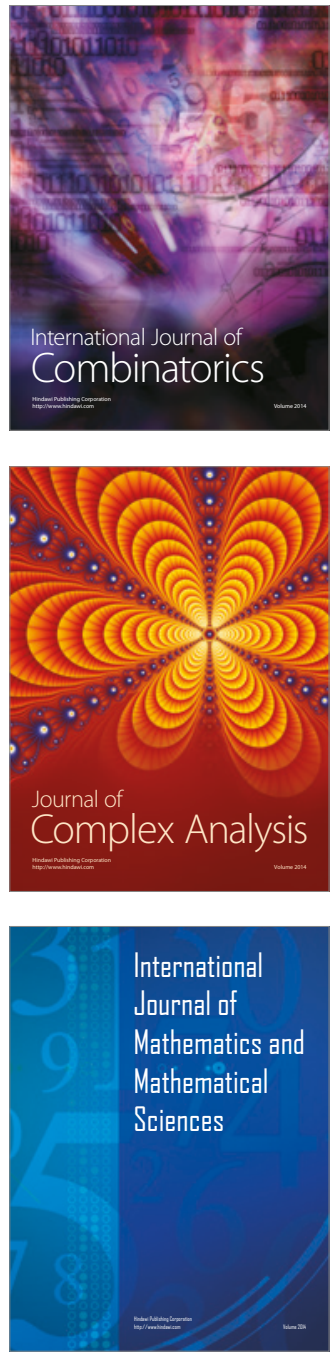
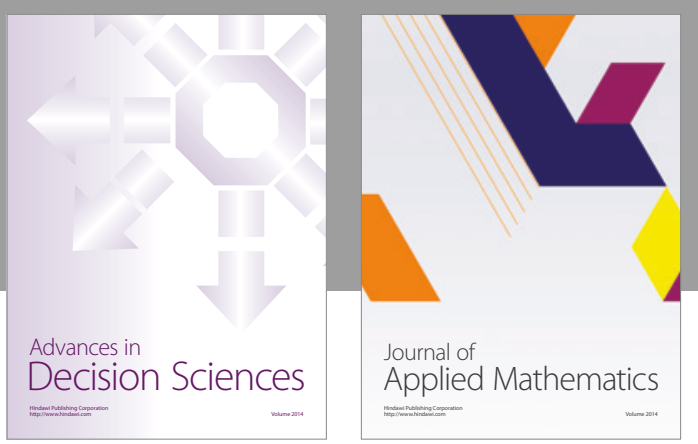

Algebra

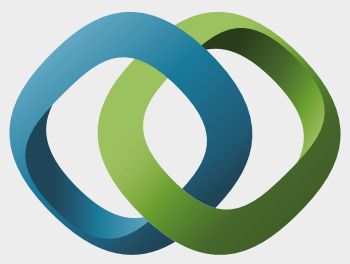

\section{Hindawi}

Submit your manuscripts at

https://www.hindawi.com
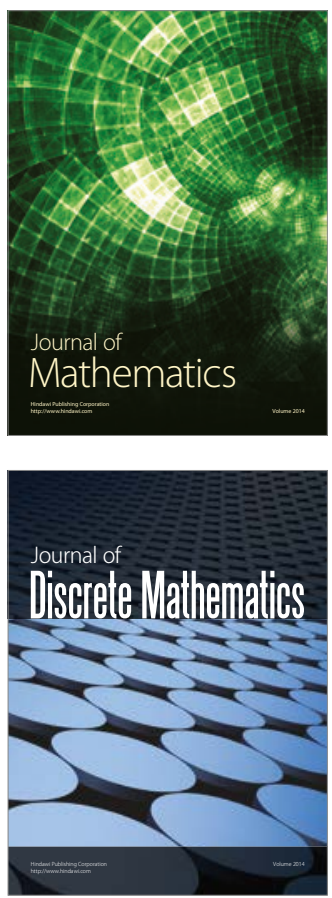

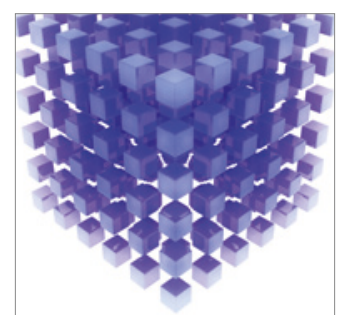

Mathematical Problems in Engineering
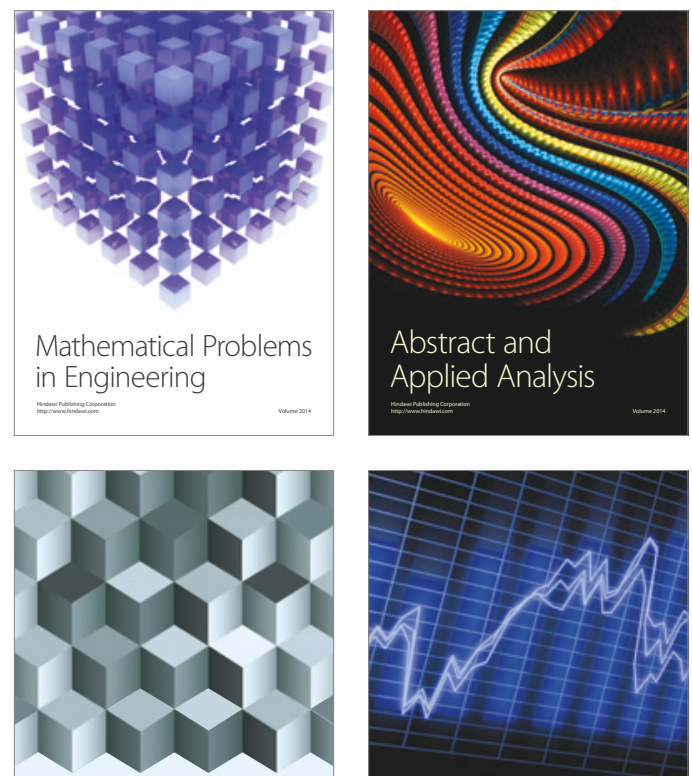

Journal of

Function Spaces

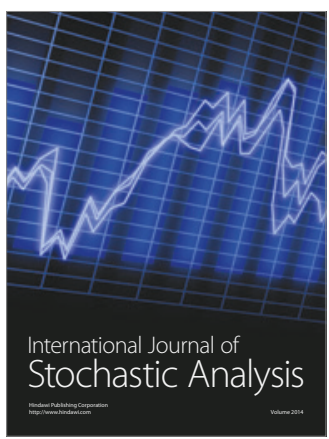

Probability and Statistics
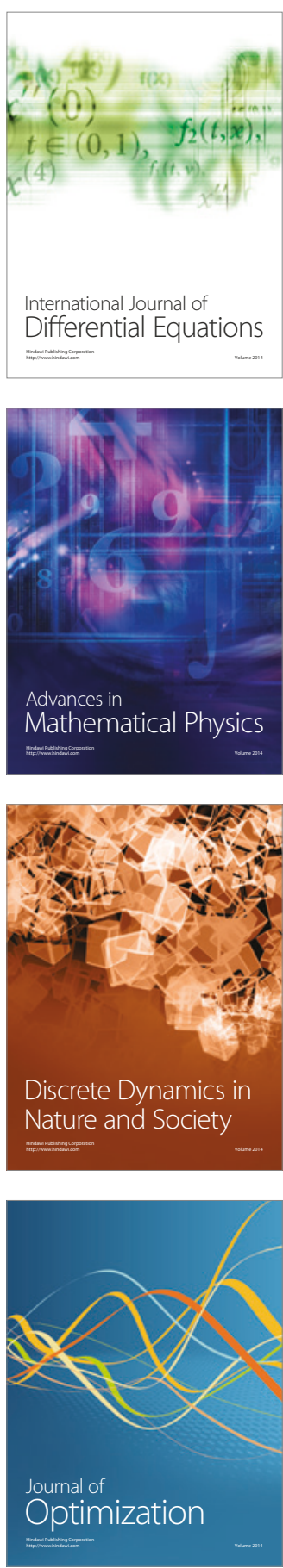